\title{
Cinética da secagem de clones de café (Coffea canephora Pierre) em terreiro de chão batido
}

\author{
Osvaldo RESENDE ${ }^{1}$, Silvestre RODRIGUES², Valdiney Cambuy SIQUEIRA ${ }^{3}$, Renan Vieira ARCANJO ${ }^{4}$
}

RESUMO

Objetivou-se com o presente trabalho estudar a cinética da secagem de quatro clones de café da espécie Coffea canephora submetidos à secagem em terreiro de chão batido, bem como ajustar diferentes modelos matemáticos aos valores experimentais selecionando aquele que melhor representa o fenômeno em estudo. Foram utilizados frutos de café dos clones: Cpafro 194, Cpafro 193, Cpafro 167 e Cpafro180, colhidos com os teores de água iniciais de 1,20; 1,32; 1,51 e 1,46 (decimal base seca (b.s.)), respectivamente. A secagem prosseguiu em terreiro de chão batido até que o produto atingisse o teor de água de 0,137 ; 0,133; 0,142 e 0,140 (decimal b.s.) respectivamente para os clones Cpafro 194, Cpafro 193, Cpafro 167 e Cpafro 180. Aos dados experimentais foram ajustados dez modelos matemáticos citados na literatura específica e utilizados para representação do processo de secagem de produtos agrícolas. Baseando-se em parâmetros estatísticos, conclui-se que os modelos Verma, Dois Termos e Aproximação da Difusão foram adequados para representação da secagem dos quatro clones de café analisados, e além destes, para o clone Cpafro 167, os modelos Thompson, Page, Newton, Logarítmico, Henderson e Pabis e Exponencial de Dois Termos também se mostraram satisfatórios na descrição do fenômeno; já o tempo necessário para a secagem em terreiro de chão batido dos clones de café Cpafro 194, Cpafro 193, Cpafro 167 e Cpafro 180 foi de 189,5 h.

PALAVRAS-CHAVE: modelagem matemática, teor de água, processamento, pós-colheita.

\section{Kinetics of coffee berry clones drying (Coffea canephora Pierre) in ground pavement}

\section{ABSTRACT}

The objective of this work was to study the drying kinetics of four coffee berry clones Coffea canephora species dried in ground pavement and to fit different mathematical models to different experimental data selecting the one that best represents the phenomenon. Have been used coffee berry of clones: Cpafro 194, Cpafro 193, Cpafro 167 and Cpafro 180, harvested at moisture content of $1.20 ; 1.32 ; 1.51$ e 1.46 (decimal d.b.), respectively. The drying continued in ground pavement until achieved the moisture content of 0.13 (decimal d.b.). Ten mathematical models cited in the literature were fitted to the experimental data in order to represent the drying process. Based on the statistical parameters it can be concluded that the Verma, Dois Termos and Aproximação da Difusão models represent well the drying of four coffee berry clones, and beyond these for the clone Cpafro 167, the Thompson, Page, Newton, Logarítmico, Henderson e Pabis and Exponencial de Dois Termos models also shown in satisfactory description of the phenomenon; the drying time in ground pavement of coffee berry clones Cpafro 194, Cpafro 193, Cpafro 167 and Cpafro 180, until the moisture content of 0.13 (decimal d.b.) was 189.5 hours.

KEYWORDS: coffee berry, clones, mathematical model, moisture content.

\footnotetext{
1 Instituto Federal de Educação, Ciência e Tecnologia Goiano, Email: osvresende@yahoo.com.br

2 Universidade Federal de Sergipe, Email: silvestrerod@yahoo.com.br

${ }^{3}$ Instituto Federal de Educação, Ciência e Tecnologia Goiano, Email: v.cambuy@hotmail.com

${ }^{4}$ Universidade Federal de Rondônia, Email: renan_v_arcanjo@hotmail.com
} 


\section{INTRODUÇÃO}

A secagem representa uma das etapas mais importantes no processamento pós-colheita do café. A conservação pela secagem baseia-se no fato de que tanto os microrganismos como as enzimas e todo mecanismo metabólico necessitam de água para suas atividades. Com a reduçáo da quantidade de água disponível, até níveis seguros para armazenagem, reduzse a atividade de água e a velocidade das reaçôes químicas no produto, bem como o desenvolvimento de microrganismos (Christensen e Kaufmann, 1974).

$\mathrm{Na}$ secagem em terreiros, ou com ar natural, o produto é retirado da planta e depositado em um pátio previamente preparado, denominado terreiro, sendo a energia utilizada para remoçâo do teor de água a radiaçáo solar. A secagem em terreiros é possível quando ocorre baixa umidade relativa do ar e pouca nebulosidade existente em diversas regióes do Brasil durante o período de colheita. A principal vantagem desse método é a economia de energia, porém apresenta o inconveniente de exigir extensas áreas e depender dos fatores climáticos, que, sendo desfavoráveis, retardam o processo favorecendo a ocorrência de fermentaçóes indesejáveis, comprometendo a qualidade do produto (Campos, 1998; Silva e Berbert, 1999).

No Brasil ainda é predominante a secagem de café em terreiros, os quais podem ser construídos de concreto, asfalto, tijolos, chão batido, leito suspenso e lama asfáltica. O produto é espalhado sobre a superfície e revolvido periodicamente. Quando o café atinge o estádio de meia seca, aproximadamente 30 \% (base úmida (b.u.)), recomenda-se amontoá-lo e cobrilo quando as condiçôes não são adequadas para a secagem.

No desenvolvimento e aperfeiçoamento de equipamentos utilizados para a secagem de grãos é de fundamental importância a simulação e a obtenção de informaçôes teóricas a respeito do comportamento de cada produto durante a remoção de água. Para a simulação, cujo princípio se fundamenta na secagem de sucessivas camadas delgadas do produto, utiliza-se um modelo matemático que representa, satisfatoriamente, sua perda de água durante o período de secagem (Berbert et al., 1995, Giner e Mascheroni, 2002).

As curvas de secagem em camada delgada variam com a espécie, variedade, condiçóes ambientais, métodos de preparo pós-colheita, entre outros fatores. Nesse sentido, diversos modelos matemáticos têm sido utilizados para descrever o processo de secagem de produtos agrícolas, embora, na maioria das vezes, as relaçôes semi-empíricas e empíricas têmse mostrado como melhores opçóes para predizer a secagem de grãos e sementes, apesar de sua validade estar restrita às condições sob as quais os dados experimentais foram obtidos (Brooker et al., 1992).
Estes modelos, geralmente, são influenciados por variáveis externas ao produto, como a temperatura e a umidade relativa do ar de secagem. Entretanto, não fornecem indicaçóes sobre os fenômenos de transporte de energia e de água no interior dos gráos e consideram que todo o processo de secagem ocorre somente no período de taxa decrescente.

As equaçóes semi-empíricas têm como base a lei de resfriamento de Newton para transferência de calor por convecção, presumindo-se que, durante a secagem, as condiçôes sejam isotérmicas e que a transferência de água seja restrita à superfície do produto (Incropera e Dewitt, 1992). Segundo Mohapatra e Rao (2005), dentre os modelos empíricos de secagem em camada fina, os mais utilizados são: Exponencial, Henderson e Pabis, Wang e Sing, Dois termos, Lewis, Page, Thompson.

Recentemente têm sido realizados inúmeros trabalhos com o objetivo de identificar as características de diversos produtos agrícolas durante a secagem como: trigo (Sun e Woods, 1994; Goneli et al., 2007), feijão (Corrêa et al., 2007; Resende et al., 2007), arroz em casca (Basunia e Abe, 2001; Resende et al., 2005), uva (Ramos et al., 2004; Ramos et al., 2005), pimenta (Kaymak-Ertekin, 2002; Akpinar et al., 2003), pêra (Lahsasni et al., 2004), trigo parbolizado (Mohapatra e Rao, 2005), tomate (Doymaz, 2007a), café em coco (Corrêa et al., 2006; Resende et al., 2007; Borém et al., 2008; Resende et al., 2009) e abóbora (Doymaz, 2007b), dentre outros.

Diante da limitaçấo de informaçôes teóricas a respeito da secagem do café da espécie Coffea Canephora, objetivou-se com o presente trabalho estudar a cinética da secagem de quatro clones de café submetidos à secagem em terreiro de cháo batido, bem como ajustar diferentes modelos matemáticos aos valores experimentais selecionando aquele que melhor representa o fenômeno em estudo.

\section{MATERIAL E MÉTODOS}

Foram utilizados frutos de café (Coffea canephora Pierre) dos clones: Cpafro 194, Cpafro 193, Cpafro 167 e Cpafro180, procedentes de lavoura situada na área experimental de clones da Embrapa em Rolim de Moura, RO.

O produto foi colhido, manualmente, pelo sistema de derriça no pano, com os teores de água iniciais de 1,$20 ; 1,32$; 1,51 e 1,46 (decimal b.s.) para os clones Cpafro 194, Cpafro 193, Cpafro 167 e Cpafro 180, respectivamente. O teor de água em base seca refere-se a relaçẫo entre a quantidade de água presente no produto e a sua massa seca.

Posteriormente à colheita, $10 \mathrm{~kg}$ de cada um dos clones de café foram submetidos a secagem em terreiro de chão batido em 3 repetiçóes, sendo espalhados em camadas de $3 \mathrm{~cm}$ de espessura e revolvidos periodicamente ao longo do dia. 
A secagem prosseguiu até que o produto atingisse o teor de água de 0,$137 ; 0,133 ; 0,142$ e 0,140 (decimal b.s.) respectivamente para os clones Cpafro 194, Cpafro 193, Cpafro 167 e Cpafro 180.

Os teores de água do produto ao longo da secagem foram acompanhados diariamente e determinados pelo método da estufa, $105 \pm 1^{\circ} \mathrm{C}$, durante $24 \mathrm{~h}$, em três repetiçóes (Brasil, 1992).

Os dados de temperatura e umidade relativa do ar de secagem foram obtidos por meio de uma estação climatológica de referência do Instituto Nacional de Pesquisas Espaciais (INPE), situada a $100 \mathrm{~m}$ do local de secagem e a temperatura da massa de café foi monitorada diariamente, por meio de sonda termométrica.

Para a avaliação da secagem dos clones de café, procedeuse à determinaçáo da taxa de redução de água do produto de acordo com a seguinte expressão:

$$
\text { TRA }=\frac{M a_{0}-M a_{i}}{M s .\left(t_{i}-t_{0}\right)}
$$

em que:

TRA : taxa de redução de água $\left(\mathrm{kg} \mathrm{kg}^{-1} \mathrm{~h}^{-1}\right)$;

$\mathrm{Ma}_{0}$ : massa de água total anterior $(\mathrm{kg})$;

$\mathrm{Ma}_{\mathrm{i}}$ : massa de água total atual $(\mathrm{kg})$;

Ms : matéria seca $(\mathrm{kg})$;

$\mathrm{t}_{0}$ : tempo total de secagem anterior $(\mathrm{h})$;

$\mathrm{t}_{\mathrm{i}}$ : tempo total de secagem atual $(\mathrm{h})$.

Devido a inexistência de isotermas de sorção para os clones de café analisados, o teor de água de equilíbrio dos frutos foi calculado utilizando-se a Equação 2 proposta por Afonso Júnior (2001) para o café da espécie Coffea arabica, expressa da seguinte forma:

$\mathrm{U}_{\mathrm{e}}^{*}=\left(2,9636+0,053 \cdot \mathrm{T}-10,7837 \cdot \mathrm{UR}^{4,5136}\right)^{-1,6503}$

em que:

$\mathrm{U}_{\mathrm{e}}^{*}$ : teor de água de equilíbrio do produto (decimal b.s.);

$\mathrm{T}$ : temperatura do ar de secagem $\left({ }^{\circ} \mathrm{C}\right)$;

UR : umidade relativa do ar de secagem (decimal).

Desta forma, de acordo com esta aproximação, obteve-se o valor do teor de água de equilíbrio de 0,0899 (decimal b.s.).

Para a determinação das razóes de umidade do café durante a secagem em terreiro de chão batido, utilizou-se a Expressão 3:

$$
\mathrm{RU}=\frac{\mathrm{U}^{*}-\mathrm{U}_{\mathrm{e}}^{*}}{\mathrm{U}_{\mathrm{e}}^{*}-\mathrm{U}_{\mathrm{i}}^{*}}
$$

em que:

RU : Razão de umidade do produto (adimensional);

$\mathrm{U}^{*}$ : teor de água do produto (decimal b.s.);
$\mathrm{U}_{\mathrm{i}}^{*}$ : teor de água inicial do produto (decimal b.s.);

Aos dados experimentais da secagem do café foram ajustados dez modelos matemáticos freqüentemente utilizados para representação da secagem de produtos agrícolas (Akpinar et al., 2003; Ertekin e Yaldiz, 2004; Lahsasni et al., 2004; Corrêa et al., 2006; Corrêa et al., 2007; Doymaz, 2007a; Doymaz, 2007b), cujas expressóes estão apresentadas na Tabela 1.

Tabela 1 - Modelos matemáticos utilizados para predizer o fenômeno de secagem de produtos agrícolas.

\begin{tabular}{lcc}
\hline Designação do modelo & \multicolumn{1}{c}{ Modelo } \\
\hline $\mathrm{RU}=1+\mathrm{at}+\mathrm{bt}^{2}$ & Wang e Singh \\
$\mathrm{RU}=\mathrm{a} \cdot \exp (-\mathrm{k} \cdot \mathrm{t})+(1-\mathrm{a}) \exp \left(-\mathrm{k}_{1} \cdot \mathrm{t}\right)$ & Verma \\
$\mathrm{RU}=\exp \left[\left[-\mathrm{a}-\left(\mathrm{a}^{2}+4 \cdot \mathrm{b} \cdot \mathrm{t}\right)^{0,5}\right] / 2 \cdot \mathrm{b}\right]$ & Thompson \\
$\mathrm{RU}=\exp \left(-\mathrm{k} \cdot \mathrm{t}^{\mathrm{n}}\right)$ & Page \\
$\mathrm{RU}=\exp (-\mathrm{k} \cdot \mathrm{t})$ & Newton \\
$\mathrm{RU}=\mathrm{a} \cdot \exp (-\mathrm{k} \cdot \mathrm{t})+\mathrm{c}$ & Logarítmico \\
$\mathrm{RU}=\mathrm{a} \cdot \exp (-\mathrm{k} \cdot \mathrm{t})$ & Henderson $\mathrm{e}$ \\
$\mathrm{RU}=\mathrm{a} \cdot \exp (-\mathrm{k} \cdot \mathrm{t})+(1-\mathrm{a})$ \\
$\mathrm{RU}=\mathrm{e} \cdot \exp (-\mathrm{exp}(-\mathrm{a} \cdot \mathrm{t})$ & Exponencial de \\
$\mathrm{RU}=\mathrm{a} \cdot \exp (-\mathrm{k} \cdot \mathrm{t})+\mathrm{b})+\left(1-\exp \left(-\mathrm{k}_{1} \cdot \mathrm{t}\right)\right.$ & Dois Termos \\
\hline
\end{tabular}

em que,

$\mathrm{t}$ : tempo de secagem $(\mathrm{s})$;

$\mathrm{k}, \mathrm{k}_{0}, \mathrm{k}_{1}$ : constantes de secagem $\left(\mathrm{s}^{-1}\right)$;

a, b, c, n : coeficientes dos modelos.

Para o ajuste dos modelos matemáticos foi realizada análise de regressão linear e não linear, pelo método Gauss Newton, utilizando-se o software Statistica $5.0^{\circledR}$. Para verificar o grau de ajuste de cada modelo considerou-se a significância do coeficiente de regressão pelo teste $\mathrm{t}$, adotando o nível de $5 \%$ de probabilidade, a magnitude do coeficiente de determinação $\left(\mathrm{R}^{2}\right)$, a magnitude do erro médio relativo $(\mathrm{P})$ e do erro médio estimado (SE) e a verificação do comportamento da distribuição dos resíduos. $\mathrm{O} \mathrm{R}^{2}$ não é uma ferramenta estatística confiável para verificar o grau de ajuste de modelos não lineares; entretanto,_normalmente, segue a tendência de ser maior para os melhores modelos. $\mathrm{O}$ erro médio relativo e o erro médio estimado, para cada um dos modelos, foram calculados conforme as Expressôes 14 e 15:

$$
\mathrm{P}=\frac{100}{\mathrm{~N}} \sum \frac{|\mathrm{Y}-\hat{\mathrm{Y}}|}{\mathrm{Y}} \quad \text { (14) } \mathrm{SE}=\sqrt{\frac{\sum(\mathrm{Y}-\hat{\mathrm{Y}})^{2}}{\mathrm{GLR}}}
$$


em que:

Y : valor observado experimentalmente;

$\hat{\mathrm{Y}}$ : valor calculado pelo modelo;

$\mathrm{N}$ : número de observaçôes experimentais;

GRL : graus de liberdade do modelo (número de observaçóes menos o número de parâmetros do modelo).

\section{RESULTADOS E DISCUSSÃO}

De acordo com o monitoramento das condiçôes climáticas, os valores médios da temperatura e da umidade relativa do ar durante o período de secagem foram de 26,3 \pm $5,0{ }^{\circ} \mathrm{C}$ e $63,3 \pm 25,0 \%$, respectivamente. Já a temperatura da massa de grãos medida diariamente às 14:00 h, apresentou variação entre 31,0 e $42,0{ }^{\circ} \mathrm{C}$ para os quatro clones analisados e durante 8 dias de monitoramento.

$\mathrm{Na}$ Figura 1, são apresentados os valores médios da taxa de remoção de água dos clones de café Cpafro 194, Cpafro 193, Cpafro 167 e Cpafro 180 para a secagem em terreiro de chão batido. Verifica-se que as maiores taxas de redução de água, para os quatro clones analisados, ocorreram no início da secagem, sendo o maior valor observado para o clone Cpafro 193 com magnitude de $0,0189 \mathrm{~kg} \mathrm{~kg}^{-1} \mathrm{~h}^{-1}$. No final da secagem, a água se encontra fortemente ligada, necessitando de maior energia para a sua evaporaçáo, assim a secagem dos clones de café ocorreu mais lentamente, resultando em menores valores da taxa de reduçáo de água.

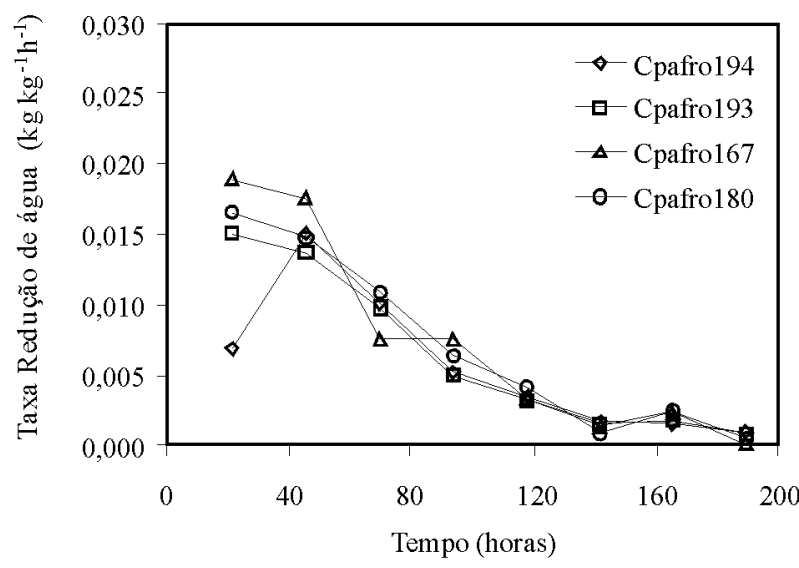

Figura 1 - Taxa de redução de água $\left(\mathrm{kg} \mathrm{kg}^{-1} \mathrm{~h}^{-1}\right)$ dos clones de café Cpafro 194, Cpafro 193, Cpafro 167 e Cpafro180 ao longo do tempo (h), durante a secagem em terreiro de chão batido.

$\mathrm{Na}$ Figura 2 estão apresentados os valores experimentais da secagem de café realizada em terreiro de chão batido para os clones Cpafro 194, Cpafro 193, Cpafro 167 e Cpafro 180.

Observa-se na Figura 2, que não foi possível detectar o período de taxa de secagem constante para as condiçóes analisadas, mesmo considerando o alto teor de água inicial

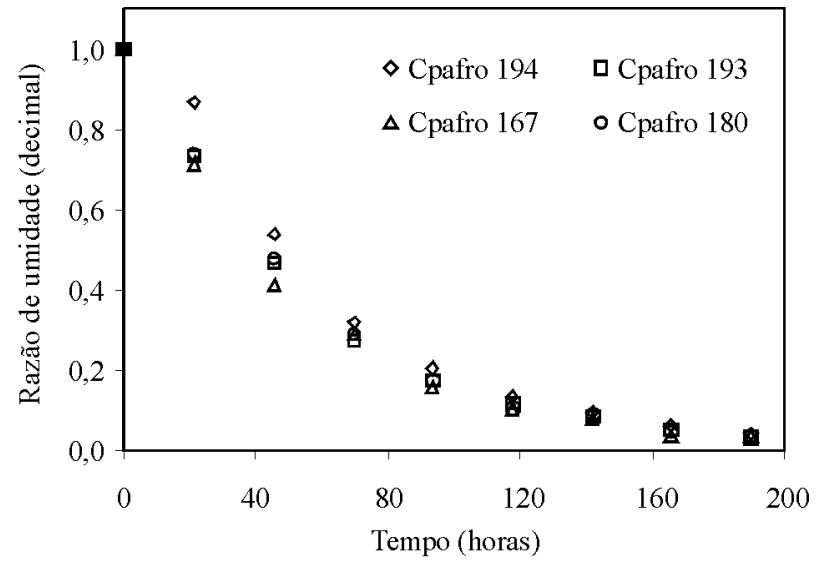

Figura 2 - Valores experimentais da razão de umidade (decimal) e do tempo de secagem (horas) para os clones Cpafro 194, Cpafro 193, Cpafro 167 e Cpafro180, durante a secagem do café.

dos cafés $(1,20 ; 1,32 ; 1,51$ e 1,46; decimal b.s.). A taxa de secagem constante é observada quando o produto se encontra completamente úmido e no início da secagem a água escoa, na fase líquida, sob um gradiente hidráulico. De acordo com Kreyger (1973), grãos e sementes, em geral, apresentam o período de secagem à taxa constante reduzido, ou inexistente, porque, nas condiçóes operacionais de secagem, as resistências às transferências de água encontram-se essencialmente no seu interior, tornando a taxa de evaporaçáo superficial acentuadamente superior à taxa de reposição de água do interior para a superfície do produto.

$\mathrm{Na}$ Tabela 2 estão apresentados os valores dos desvios padrão para o teor de água ao longo da secagem para os clones de café Cpafro 194, Cpafro 193, Cpafro 167 e Cpafro180. Verifica-se que os desvios diminuem com a redução do teor de água para os quatro clones de café analisados.

Tabela 2 - Valores dos desvios padrão para o teor de água ao longo da secagem em terreiro de chão batido para os clones de café Cpafro 194, Cpafro 193, Cpafro 167 e Cpafro 180.

\begin{tabular}{cccc}
\hline \multicolumn{4}{c}{ Desvio padrão (decimal b.s.) } \\
\hline Cpafro 194 & Cpafro 193 & Cpafro 167 & Cpafro 180 \\
0,118 & 0,052 & 0,039 & 0,040 \\
0,060 & 0,056 & 0,043 & 0,027 \\
0,028 & 0,031 & 0,005 & 0,021 \\
0,012 & 0,016 & 0,018 & 0,007 \\
0,007 & 0,011 & 0,018 & 0,009 \\
0,009 & 0,022 & 0,003 & 0,007 \\
0,004 & 0,005 & 0,009 & 0,007 \\
0,002 & 0,004 & 0,005 & 0,002 \\
0,001 & 0,004 & 0,004 & 0,001 \\
\hline
\end{tabular}


Na Tabela 3, encontram-se os parâmetros estatísticos utilizados para a comparação entre os dez modelos analisados para a secagem dos quatro clones de café da espécie Coffea canephora.

O coeficiente de determinação apesar de não ser um bom parâmetro para verificar o ajuste de modelos não-lineares, normalmente apresenta maiores valores para os modelos que melhor se ajustam aos dados experimentais. De acordo com a Tabela 3, observa-se que para os clones Cpafro 193, Cpafro 167 e Cpafro 180 os dez modelos matemáticos ajustados aos dados experimentais apresentaram coeficientes de determinação $\left(\mathrm{R}^{2}\right)$ superior a $98 \%$, indicando, de acordo com Madamba et al. (1996), uma representaçáo satisfatória do fenômeno em estudo.
Os modelos Verma, Dois Termos e Aproximação da Difusão apresentaram os menores valores do erro médio estimado para os quatro clones analisados. Para o clone Cpafro 194, os modelos de Verma, Dois Termos e Aproximaçáo da Difusão obtiveram magnitudes do erro médio relativo inferior a 10\%, indicando, de acordo com Mohapatra e Rao (2005), serem adequados para a descrição do fenômeno; para os clones Cpafro 193 e Cpafro 167 somente o modelo de Wang e Singh apresentou valor do erro médio relativo superior a $10 \%$, ratificando a sua não adequaçáo na representaçáo do fenômeno em estudo para este produto; e, finalmente, para o clone Cpafro 180, os modelos Wang e Singh, Page e Logarítmico também forneceram magnitudes do erro médio relativo superior ao estabelecido pelo referido autor, desabilitando estes modelos a satisfatória descrição da secagem do clone em questão.

Tabela 3 - Coeficientes de determinação ( $\left.R^{2}\right)$, erros médio relativo (P) e estimado (SE), e tendência de distribuição dos resíduos, para os dez modelos analisados, durante a secagem dos clones Cpafro 194, Cpafro 193, Cpafro 167 e Cpafro 180 em terreiro de chão batido.

\begin{tabular}{|c|c|c|c|c|}
\hline \multicolumn{5}{|c|}{ Cpafro 194} \\
\hline Modelo & $\mathrm{R}^{2}(\%)$ & SE (decimal) & $\mathrm{P}(\%)$ & Dist. dos resíduos \\
\hline Wang e Singh & 98,63 & 0,0450 & 16,82 & Tendenciosa \\
\hline Verma & 99,93 & 0,0113 & 7,51 & Aleatória \\
\hline Thompson & 97,42 & 0,0618 & 18,34 & Tendenciosa \\
\hline Page & 99,29 & 0,0323 & 18,07 & Tendenciosa \\
\hline Newton & 97,42 & 0,0578 & 18,33 & Tendenciosa \\
\hline Logarítmico & 98,30 & 0,0542 & 16,45 & Tendenciosa \\
\hline Henderson e Pabis & 98,01 & 0,0542 & 13,64 & Tendenciosa \\
\hline Exponencial de Dois Termos & 97,42 & 0,0618 & 18,75 & Tendenciosa \\
\hline Dois Termos & 99,93 & 0,0124 & 7,51 & Aleatória \\
\hline Aproximação da Difusão & 99,93 & 0,0113 & 7,51 & Aleatória \\
\hline \multicolumn{5}{|c|}{ Cpafro 193} \\
\hline Wang e Singh & 98,90 & 0,0382 & 31,06 & Tendenciosa \\
\hline Verma & 99,94 & 0,0099 & 6,26 & Aleatória \\
\hline Thompson & 99,62 & 0,0224 & 6,25 & Tendenciosa \\
\hline Page & 99,86 & 0,0137 & 9,55 & Tendenciosa \\
\hline Newton & 99,62 & 0,0209 & 6,24 & Tendenciosa \\
\hline Logarítmico & 99,71 & 0,0212 & 8,31 & Tendenciosa \\
\hline Henderson e Pabis & 99,69 & 0,0204 & 5,15 & Tendenciosa \\
\hline Exponencial de Dois Termos & 99,62 & 0,0225 & 6,32 & Tendenciosa \\
\hline Dois Termos & 99,94 & 0,0108 & 6,26 & Aleatória \\
\hline Aproximação da Difusão & 99,94 & 0,0099 & 6,26 & Aleatória \\
\hline \multicolumn{5}{|c|}{ Cpafro 167} \\
\hline Wang e Singh & 98,40 & 0,0458 & 32,70 & Tendenciosa \\
\hline Verma & 99,86 & 0,0147 & 8,02 & Aleatória \\
\hline Thompson & 99,72 & 0,0192 & 9,00 & Aleatória \\
\hline Page & 99,80 & 0,0161 & 9,04 & Aleatória \\
\hline Newton & 99,71 & 0,0181 & 8,99 & Aleatória \\
\hline Logarítmico & 99,74 & 0,0198 & 9,30 & Aleatória \\
\hline Henderson e Pabis & 99,74 & 0,0184 & 8,98 & Aleatória \\
\hline Exponencial de Dois Termos & 99,71 & 0,0195 & 9,00 & Aleatória \\
\hline Dois Termos & 99,86 & 0,0161 & 8,02 & Aleatória \\
\hline Aproximação da Difusão & 99,85 & 0,0150 & 8,01 & Aleatória \\
\hline
\end{tabular}


Tabela 3 - Continuação.

\begin{tabular}{lcccc}
\hline \multicolumn{7}{c}{ Cpafro 180} & & & \\
\hline Wang e Singh & 99,19 & 0,0329 & 26,50 & Tendenciosa \\
\hline Verma & 99,92 & 0,0112 & 6,91 & Aleatória \\
\hline Thompson & 99,51 & 0,0256 & 9,63 & Tendenciosa \\
\hline Page & 99,87 & 0,0131 & 10,75 & Tendenciosa \\
Newton & 99,51 & 0,0240 & 9,62 & Tendenciosa \\
Logarítmico & 99,65 & 0,0233 & 11,76 & Tendenciosa \\
Henderson e Pabis & 99,60 & 0,0233 & 8,69 & Tendenciosa \\
\hline Exponencial de Dois Termos & 99,50 & 0,0258 & 9,85 & Tendenciosa \\
Dois Termos & 99,92 & 0,0123 & 6,91 & Aleatória \\
Aproximação da Difusão & 99,92 & 0,0113 & 6,83 & Aleatória \\
\hline
\end{tabular}

Verifica-se ainda na Tabela 3, que os modelos Verma, Dois Termos e Aproximaçấo da Difusão obtiveram para a modelagem da secagem dos clones Cpafro 194, Cpafro 193 e Cpafro 180, uma distribuição aleatória dos resíduos resultando, assim, em um ajuste satisfatório aos dados experimentais. Para o clone Cpafro 167 todos os modelos, exceto o Wang e Singh, apresentaram a distribuição dos resíduos aleatória, indicando a adequação dos modelos em representar a secagem com ar natural em terreiro de chão batido, e sob as condiçóes experimentadas.

$\mathrm{Na}$ Figura 3 estão apresentadas as ilustraçóes da distribuição dos resíduos (Aleatória para o modelo de Verma e tendenciosa para o modelo de Wang e Singh), durante a modelagem da secagem do clone Cpafro 194.

De acordo com os parâmetros estatísticos avaliados, os modelos Verma, Dois Termos e Aproximaçấo da Difusão foram adequados para representaçáo da secagem dos clones de café Cpafro 194, Cpafro 193 e Cpafro 180, e além destes, para o clone Cpafro 167, os modelos Thompson, Page, Newton, Logarítmico, Henderson e Pabis e Exponencial de Dois Termos também se mostraram satisfatórios na descrição do fenômeno. Estes resultados corroboram os obtidos por Resende et al. (2009), que verificaram que os modelos Verma, Dois Termos e Aproximação da Difusão descreveram satisfatoriamente a secagem em terreiro de concreto destes mesmos clones de café da espécie Coffea canephora, Cpafro 194, Cpafro 193, Cpafro 167 e Cpafro 180.

Para Corrêa et al. (2006), o modelo Verma representou satisfatoriamente o fenômeno de secagem dos frutos de café, cultivar Mundo Novo, da espécie Coffea arabica. Doymaz (2007b) verificou que o modelo de Verma descreveu adequadamente a secagem de fatias de abóbora.

Babalis et al. (2006) e Mohamed et al. (2008) notaram que o modelo de Dois Termos obteve melhor ajuste aos dados experimentais da secagem de figo e algas marinhas (Gelidium sesquipedale), respectivamente.
A) Aleatória

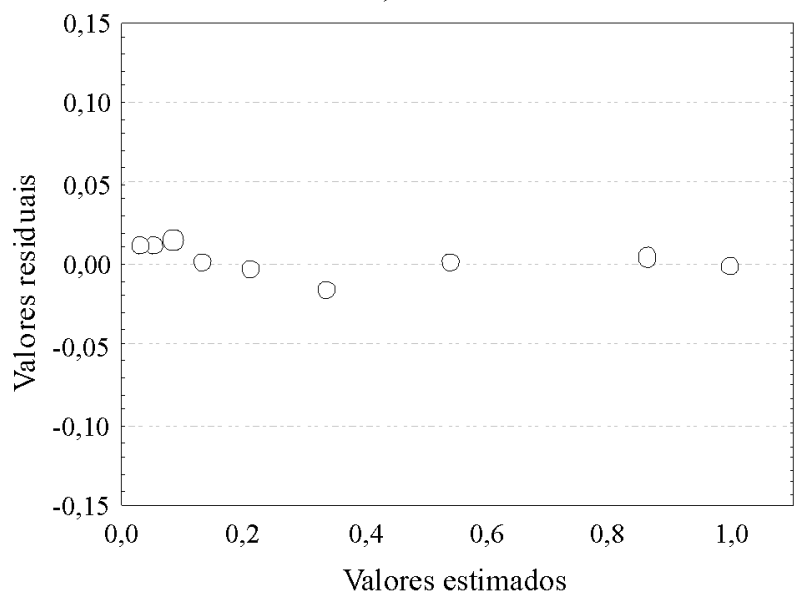

B) Aleatória

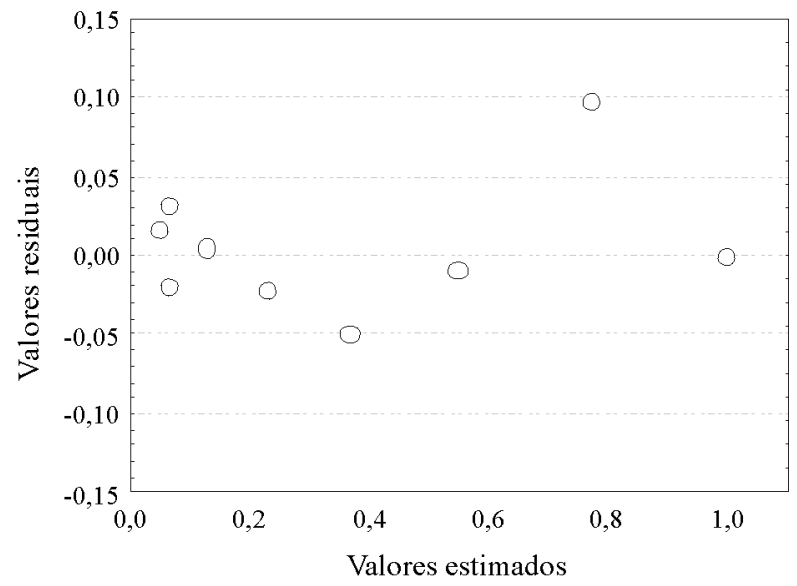

Figura 3 - Ilustração da distribuição dos resíduos: A) Aleatória para o modelo de Verma; B) Tendenciosa para o modelo de Wang e Singh; durante a modelagem da secagem do clone Cpafro 194. 
Na Tabela 4 estão os coeficientes dos modelos Verma, Dois Termos e Aproximaçáo da Difusão ajustados para os quatro clones analisados durante a secagem em terreiro de chão batido.

A seleção e recomendação do melhor modelo se fundamentaram na simplicidade e no número de coeficientes. Dentre os três modelos que apresentaram bons ajustes aos quatro clones de café, o modelo de Verma foi selecionado para representar o fenômeno de secagem dos clones de café devido a sua maior simplicidade em comparação aos demais modelos selecionados.

Na Figura 4, estão ilustrados os teores de água observados e estimados pelo modelo de Verma, para os clones Cpafro 194, Cpafro 193, Cpafro 167 e Cpafro 180, durante a secagem do café. O modelo original de Verma estima o valor da razão de umidade que, por meio de uma inversão matemática, pode-se obter diretamente o teor de água do produto. Nota-se, por meio da correspondência entre os valores experimentais e estimados, que o modelo de Verma descreve adequadamente o fenômeno de secagem dos clones de café estudados.

Verifica-se na Figura 4, que mesmo com os teores de água iniciais dos clones de café sendo distintos, o tempo necessário para que o produto atingisse o teor de água de 0,$137 ; 0,133$; 0,142 e 0,140 (decimal b.s.) foi de aproximadamente 189,5 h, respectivamente para os clones Cpafro 194, Cpafro 193, Cpafro 167 e Cpafro 180. Ao final da secagem, as taxas de remoçáo de água diminuíram e as discrepâncias entre os valores dos teores de água dos clones, consequentemente, reduziram. Pode-se inferir que este fato, possivelmente, deveu-se à tendência dos clones atingirem o mesmo equilíbrio higroscópico com as condiçôes médias do ar de secagem.

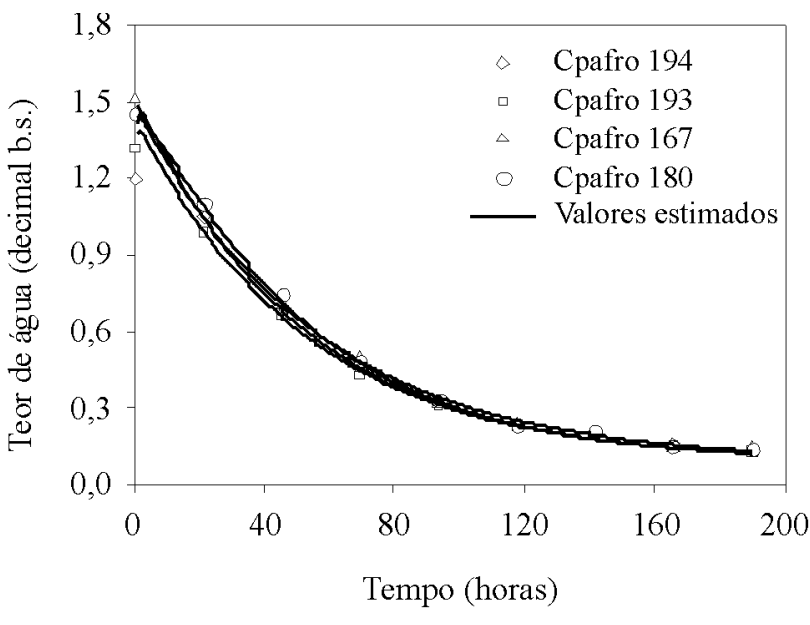

Figura 4 - Valores experimentais e estimados pelo modelo de Verma (decimal b.s.) dos teores de água ao longo do tempo de secagem (h), para os clones de café Cpafro 194, Cpafro 193, Cpafro 167 e Cpafro 180.

Tabela 4 - Coeficientes ajustados para os modelos Verma, Dois Termos e Aproximação da Difusão, durante a secagem dos clones Cpafro 194, Cpafro 193 Cpafro 167 e Cpafro 180 em terreiro de chão batido.

\begin{tabular}{|c|c|c|c|c|c|}
\hline \multirow[b]{2}{*}{ Modelos } & \multirow[b]{2}{*}{ Coeficientes } & \multicolumn{4}{|c|}{ Clones } \\
\hline & & Cpafro 194 & Cpafro 193 & Cpafro 167 & Cpafro180 \\
\hline \multirow{3}{*}{ Verma } & a & $-0,316$ & $-0,111$ & $-0,078$ & $-0,126$ \\
\hline & k & 0,100 & 0,100 & 0,100 & 0,100 \\
\hline & $\mathrm{k}_{1}$ & $5,46 \times 10^{-6}$ & $5,35 \times 10^{-6}$ & $5,49 \times 10^{-6}$ & $5,36 \times 10^{-6}$ \\
\hline \multirow{4}{*}{ Dois Termos } & a & $-0,316$ & $-0,111$ & $-0,078$ & $-0,126$ \\
\hline & $\mathrm{k}_{0}$ & 0,010 & 0,010 & 0,010 & 0,010 \\
\hline & $b$ & 1,316 & 1,111 & 1,078 & 1,126 \\
\hline & $\mathrm{k}_{1}$ & $5,46 \times 10^{-6}$ & $5,35 \times 10^{-6}$ & $5,49 \times 10^{-6}$ & $5,36 \times 10^{-6}$ \\
\hline \multirow{3}{*}{ Aproximação da Difusão } & a & $-0,316$ & $-0,111$ & $-0,095$ & $-0,126$ \\
\hline & k & $7,50 \times 10^{-3}$ & $6,54 \times 10^{-3}$ & $9,32 \times 10^{-3}$ & $5,51 \times 10^{-3}$ \\
\hline & $\mathrm{b}$ & $7,28 \times 10^{-4}$ & $8,18 \times 10^{-4}$ & $5,97 \times 10^{-4}$ & $9,70 \times 10^{-4}$ \\
\hline
\end{tabular}




\section{CONCLUSÕES}

Baseando-se em parâmetros estatísticos, conclui-se que os modelos Verma, Dois Termos e Aproximação da Difusão foram adequados para representaçáo da secagem dos quatro clones de café analisados; já o tempo necessário para a secagem em terreiro de chão batido dos clones de café Cpafro 194, Cpafro 193, Cpafro 167 e Cpafro 180, até o teor de água de 0,13 (decimal b.s.) foi de, aproximadamente $189,5 \mathrm{~h}$.

\section{BIBLIOGRAFIA CITADA}

Afonso Júnior, P.C. 2001. Coffee physical, physiological aspects and coffee quality in function of drying and storage. Tese de Doutorado, Universidade Federal de Viçosa, Viçosa, Minas Gerais. 384pp (in Portuguese).

Akpinar, E.K.; Bicer, Y.; Yildiz, C. 2003. Thin layer drying of red pepper. Journal of Food Engineering, 59(1): 99-104.

Babalis, J.S.; Papanicolaou, E.; Kyriakis, N.; Belessiotis, V.G. 2006. Evaluation of thin-layer drying models for describing drying kinetics of figs (Ficus carica). Journal of Food Engineering, 75(2): 205-214.

Basunia, M.A., Abe, T. 2001. Moisture desorption isotherms of medium-grain rough rice. Journal of Stored Products Research, 37(3): 205-219.

Berbert, P.A.; Queiroz, D.M.; Silva, J.S.; Pinheiro Filho, J.B. 1995. Simulation of coffee drying in a fixed bed with periodic airflow reversal. Journal of Agricultural Engineering Research, 60(3): 167-173.

Borém, F. M.; Marques, E. R.; Alves, E. 2008. Ultrastructural analysis of drying damage in parchment Arabica coffee endosperm cells. Biosystems Engineering. 99(1):62-66.

Brasil, Ministério da Agricultura e Reforma Agrária. 1992. Secretaria Nacional de Defesa Agropecuária. Seeds analysis standard. Brasília, $365 p p$ (in Portuguese).

Brooker, D.B.; Bakker-Arkema, F.W.; Hall, C.W. 1992. Drying and storage of grains and oilseeds. Westport, The AVI Publishing Company. 450 pp.

Campos, A.T.; Melo, E.C.; Silva, J.S.E. 1999. Development and analysis of a fixed-bed dryer prototype for coffee (Coffea Arabica L.) with a mechanical revolving sistem. Revista Brasileira de Armazenamento. 24(2):37-41 (in Portuguese, with abstract in English).

Christensen, C.M.; Kaufmann, H.H. 1974. Microflora. In: Christensen, C.M. (Ed) Storage of cereal grain and their products. St. Paul: American Association of Cereal Chemists. p.158-192.

Corrêa, P.C.; Resende, O.; Martinazzo, A.P.; Goneli, A.L.D.; Botelho, F.M. 2007. Mathematical modelling for describing the drying process of the edible bean (Phaseolus vulgaris L.) in thin layers. Engenharia Agricola, 27(2): 501-510 (in Portuguese, with abstract in English).

Corrêa, P.C.; Resende, O.; Ribeiro, D.M. 2006. Drying characteristics and kinetics of coffee berry. Revista Brasileira de Produtos Agroindustriais, 8(1): 1-10.
Doymaz, I. 2007a. Air-drying characteristics of tomatoes. Journal of Food Engineering, 78(4): 1291-1297.

Doymaz, I. 2007b. The kinetics of forced convective air-drying of pumpkin slices. Journal of Food Engineering, 79(1): 243-249.

Ertekin, C.; Yaldiz, O. 2004. Drying of eggplant and selection of a suitable thin layer drying model. Journal of Food Engineering, 63(3): 349-359.

Giner, S.A.; Mascheroni, R.H. 2002. Diffusive drying kinetics in wheat, Part 2: applying the simplified analytical solution to experimental data. Postharvest Technology, 81(1): 85-97.

Goneli, A.L.D.; Corrêa, P.C.; Resende, O.; Reis Neto, S.A. 2007. Study of moisture diffusion in wheat grain drying. Ciência e Tecnologia de Alimentos, 27(1): 135-140 (in Portuguese, with abstract in English).

Incropera, F.P.; Dewitt, D.P. 1992. Fundamentals of heat and mass transfer. Rio de Janeiro, Editora Guanabara Koogan S.A. 380pp (in Portuguese).

Kaymak-Ertekin, F. 2002. Drying and rehydrating kinetics of green and red peppers. Journal of Food Science, 67(1): 168-175.

Kreyger, J. 1973. Practical observations on the drying of seed. Seed Science and Technology, 1: 645-670.

Lahsasni, S.; Kouhila, M.; Mahrouz, M.; Jaouhari, J.T. 2004. Drying kinetics of prickly pear fruit (Opuntia ficus indica). Journal of Food Engineering, 61(2): 173-179.

Madamba, P.S.; Driscoll, R.H.; Buckle, K.A. 1996. Thin-layer drying characteristics of garlic slices. Journal of Food Engineering, 29(1): 75-97.

Mohamed, L.A.; Kane, C.S.E.; Kouhila, M.; Jamali, A.; Mahrouz, M.; Kechaou, N. 2008. Thin layer modelling of Gelidium sesquipedale solar drying process. Energy Conversion and Management, 49(5): 940-946.

Mohapatra, D.; Rao, P.S. 2005. A thin layer drying model of parboiled wheat. Journal of Food Engineering, 66(4): 513-518.

Ramos, I.N.; Brandão, T.R.S.; Silva, C.L.M. 2005. Integrated approach on solar drying, pilot convective drying and microstructural changes. Journal of Food Engineering, 67(1-2): 195-203.

Ramos, I.N.; Silva, C.L.M.; Sereno, A.M.; Aguilera, J.M. 2004. Quantification of microstructural changes during first stage air drying of grape tissue. Journal of Food Engineering, 62(2): 159-164.

Resende, O.; Arcanjo, R.V.; Siqueira, V.C.; Rodrigues, S.; Kester, A.N.; Lima, P.P. 2007. Influence of pavement type in coffee berry clones drying (Coffea canephora Pierre) in concrete and ground yards. Revista Brasileira de Produtos Agroindustriais. 9(2): 171-178 (in Portuguese, with abstract in English).

Resende, O.; Arcanjo, R.V.; Siqueira, V.C.; Rodrigues, S. 2009. Mathematical modeling for drying coffee (Coffea canephora Pierre) berry clones in concrete yard. Acta Scientiarum. Agronomy, 31(2): 189-196 (in Portuguese, with abstract in English).

Resende, O.; Corrêa, P.C.; Goneli, A.L.D.; Martinazzo, A.P.; Ribeiro, R.M. 2005. The shrinkage in the liquid diffusion during the drying process of the rough rice. Revista Brasileira de 


\section{ACTA}

Armazenamento, 30(2): 63-171 (in Portuguese, with abstract in English).

Resende, O.; Corrêa, P.C.; Jaren, C.; Moure, A.J. 2007. Bean moisture diffusivity and drying kinetics: a comparison of the liquid diffusion model when taking into account and neglecting grain shrinkage. Spanish Journal of Agricultural Research, 5(1): 51-58.

Silva, J.S.; Berbert, P.A. 1999. Harvest, drying and storage. Viçosa, Aprenda Fácil. 145pp (in Portuguese).
Sun, D.W.; Woods, J.L. 1994. Low temperature moisture transfer characteristics of wheat in thin layers. Journal of Agricultural Engineering Research, 59(4): 273-283.

Recebido em 29/01/2008

Aceito em 13/11/2009 
